\title{
On the Non-Trivial Zeros of Dirichlet Functions
}

\author{
Dorin Ghisa \\ York University, Toronto, Canada \\ Email: dghisa@yorku.ca
}

How to cite this paper: Ghisa, D. (2021) On the Non-Trivial Zeros of Dirichlet Functions. Advances in Pure Mathematics, 11, 187-204.

https://doi.org/10.4236/apm.2021.113014

Received: February 23, 2021

Accepted: March 26, 2021

Published: March 29, 2021

Copyright $\odot 2021$ by author(s) and Scientific Research Publishing Inc. This work is licensed under the Creative Commons Attribution International License (CC BY 4.0).

http://creativecommons.org/licenses/by/4.0/ (c) (i) Open Access

\begin{abstract}
The purpose of this research is to extend to the functions obtained by meromorphic continuation of general Dirichlet series some properties of the functions in the Selberg class, which are all generated by ordinary Dirichlet series. We wanted to put to work the powerful tool of the geometry of conformal mappings of these functions, which we built in previous research, in order to study the location of their non-trivial zeros. A new approach of the concept of multiplier in Riemann type of functional equation was necessary and we have shown that with this approach the non-trivial zeros of the Dirichlet function satisfying a Reimann type of functional equation are either on the critical line, or they are two by two symmetric with respect to the critical line. The Euler product general Dirichlet series are defined, a wide class of such series is presented, and finally by using geometric and analytic arguments it is proved that for Euler product functions the symmetric zeros with respect to the critical line must coincide.
\end{abstract}

\section{Keywords}

General Dirichlet Series, Riemann Type of Functional Equation, Euler Products, Fundamental Domains, Analytic Continuation

\section{Introduction}

The fundamental domains, as defined by Ahlfors, are for the complex functions of one complex variable the similar of the intervals of monotony for the real ones, in the sense that in both cases the functions are injective there. However, for the analytic functions of one complex variable the concept of fundamental domain plays a much deeper role. Such a domain is mapped conformally by the function onto the whole complex plane with some slits. The rational functions have a finite number of fundamental domains (the same as their degree), while the transcendental ones have infinitely many fundamental domains. Their closure covers the entire complex plane.

The Dirichlet functions are meromorphic functions in the complex plane 
having an essential singular point at infinity. We have shown in previous works that every neighbourhood of an essential singular point of an analytic function contains infinitely many fundamental domains. Therefore a Dirichlet function has infinitely many fundamental domains outside any bounded region of complex plane. We found that these domains are infinite strips which can be obtained by using the pre-image of the real axis and the zeros of the derivative of the function, necessarily situated on the common boundaries of these domains. These are basic knowledge in the study of the location of non-trivial zeros of $\mathrm{Di}$ richlet functions.

The symmetric zeros with respect to the critical line are located in adjacent fundamental domains, which can be mapped conformally one onto the other such that the image of a zero is the other zero. This function can be extended to an involution on the union of the two domains and this is instrumental in using the Euler product in order to prove $\mathrm{RH}$, which is the final result of this paper.

\section{Meromorphic Continuation of General Dirichlet Series}

The Dirichlet functions are meromorphic continuations into the whole complex plane of general Dirichlet series. The study of general Dirichlet series has its origins in the works of Cahen [1] [2], Hadamard [3], Landau [4] [5], Bohr [6] [7] [8], Hardi \& Riesz [9], Kojima [10], Valiron [11] etc.

We are dealing with normalized series defined by using an arbitrary sequence of complex numbers $A=\left(1=a_{1}, a_{2}, \cdots\right)$, (the coefficients) and an increasing sequence $\Lambda=\left(0=\lambda_{1}<\lambda_{2}<\cdots\right), \lim _{n \rightarrow \infty} \lambda_{n}=\infty$, (the exponents) in the following way:

$$
\zeta_{A, \Lambda}(s)=\sum_{n=1}^{\infty} a_{n} \mathrm{e}^{-\lambda_{n} s}
$$

When $\lambda_{n}=\ln n$ we obtain the ordinary (proprement dites [11]) Dirichlet series $\sum_{n=1}^{\infty} \frac{a_{n}}{n^{s}}$. When $\lambda_{n}=n-1$, the series (1) becomes a power series of $z=\mathrm{e}^{-s}$. Vice-versa, any power series $\sum_{n=0}^{\infty}\left(z-z_{0}\right)^{n}$ can be converted into a Dirichlet series $\sum_{n=0}^{\infty} a_{n} \mathrm{e}^{-n s}$ upon the substitution $z-z_{0}=\mathrm{e}^{-s}$. The Hadamard formula $\frac{1}{R}=\limsup _{n \rightarrow \infty}\left|a_{n}\right|^{1 / n}$ giving the radius of convergence $R$ of that series shows also where the corresponding Dirichlet series is convergent, namely for $\left|\mathrm{e}^{-s}\right|<R$. If $s=\sigma+i t$, this means that the series converges for $\sigma>\ln \frac{1}{R}$ and diverges for $\sigma<\ln \frac{1}{R}$. There is an analog of this formula for arbitrary Dirichlet series (see, for example [11]) which says that the series (1) converges into a right hand half plane $\operatorname{Re} s>\sigma_{c}$ and it diverges into the left hand half plane $\operatorname{Re} s<\sigma_{c}$, where $-\infty \leq \sigma_{c} \leq+\infty$. When (1) does not converge for $s=0$, then (see [11]):

$$
\sigma_{c}=\lim \sup _{n \rightarrow \rightarrow \infty} \frac{\ln \left|\sum_{k=1}^{n} a_{k}\right|}{\lambda_{n}} \geq 0
$$


and when (1) does converge for $s=0$, then:

$$
\sigma_{c}=\lim \sup _{n \rightarrow \infty} \frac{1}{\lambda_{n+1}} \ln \left|\zeta_{A, \Lambda}(0)-\sum_{k=1}^{n} a_{k}\right|
$$

The number $\sigma_{c}$ is called the abscissa of convergence of the series. The series (1) converges uniformly on compact sets included in the half plane $\operatorname{Re} s>\sigma_{c}$ and therefore it is an analytic function there. The abscissa of absolute convergence $\sigma_{a}$ of the series (1) is by definition the abscissa of convergence of the series $\sum_{n=1}^{\infty}\left|a_{n}\right| \mathrm{e}^{-\lambda_{n} s}$. The line $\operatorname{Re} s=\sigma_{c}$ is called the line of convergence of the series, although as we will see later, there are Dirichlet series which are divergent at every point of that line. Obviously, for those series, there cannot be meromorphic continuation across the convergence line and therefore they do not generate Dirichlet functions. We deal in this paper only with series generating Dirichlet functions. We found in [12] a sufficient condition for the normalized Dirichlet series (1) to admit meromorphic continuation into the whole complex plane. It is expressed in terms of the associated series

$$
\zeta_{A, \mathrm{e}^{\Lambda}}(s)=\sum_{n=1}^{\infty} a_{n} \mathrm{e}^{-\mathrm{e}^{\lambda_{n}}}
$$

in which the exponents $\lambda_{n}$ are replaced by $\mathrm{e}^{\lambda_{n}}$. The relationship between $\zeta_{A, \Lambda}(s)$ and $\zeta_{A, \mathrm{e}^{\Lambda}}(s)$ has been studied by Cahen [1] who proved (see also [12]):

Proposition 1: If the series (1) has a finite abscissa of convergence, then the series (4) has the abscissa of convergence zero.

Analogously, if the series (1) has a finite abscissa of absolute convergence, then the series (4) has the abscissa of absolute convergence zero.

The sufficient condition found in [12] for the series (1) to admit meromorphic continuation into the whole complex plane can be relaxed as follows:

Theorem 1: Suppose that the series (1) has a finite abscissa of absolute convergence $\sigma_{a}$ and the series (4) has a discrete set of singular points on the imaginary axis in the neighborhood of the origin. Then the series (1) can be continued meromorphically into the whole complex plane.

Proof: We will use the Riemann's technique of contour integration ([13], page 214). Namely, in the integral formula of Euler Gamma function, true for $\operatorname{Re} s>1$

$$
\Gamma(s)=\int_{0}^{\infty} x^{s-1} \mathrm{e}^{-x} \mathrm{~d} x
$$

we replace $x$ by $\mathrm{e}^{\lambda_{n}} x$. Then (5) implies:

$$
\mathrm{e}^{-\lambda_{n} s}=\frac{1}{\Gamma(s)} \int_{0}^{\infty} x^{s-1} \mathrm{e}^{-\mathrm{e}^{\lambda} x} \mathrm{~d} x
$$

If we multiply here the $n$-th term by $a_{n}$ and add from $n=1$ to $\infty$ we get

$$
\zeta_{A, \Lambda}(s)=\frac{1}{\Gamma(s)} \int_{0}^{\infty} x^{s-1} \zeta_{A, \mathrm{e}^{\Lambda}}(x) \mathrm{d} x
$$

Indeed, the series $\zeta_{A, \mathrm{e}^{\Lambda}}(x)$ converges for $x>0$ by Proposition 1 , hence the 
integral (7) makes sense. On the other hand, the interchange of summation and integration was possible since the integral in (6) is absolutely convergent at both ends, the integral in (5) being absolutely convergent at both ends (see [13], page 214 ) and (6) is obtained from (5) by a change of variable conserving the limits of integration.

The function $\Gamma(s)$ can be uniquely continued to a meromorphic function into the whole complex plane, hence the formula (7) is true for $\operatorname{Re} s>\sigma_{c}$ even if $\sigma_{c}<1$. Yet, the right hand side in (7) has a meaning for all the values of $s$ for which the integral in (7) is convergent, given the fact that $\Gamma(s)$ has no zero. When that integral is convergent for $\operatorname{Re} s \leq \sigma_{c}$ the formula (7) provides a continuation of $\zeta_{A, \Lambda}(s)$. Trying to extend this continuation to the whole complex plane by proceeding as Riemann did for $\zeta(s)$ (see [13], page 214) we are confronted with a difficulty, since the integration contour used by Riemann is inappropriate in our case, $\zeta_{A \mathrm{e}^{\Lambda}}(z)$ not being defined for $\operatorname{Re} z \leq 0$. We can avoid this inconvenience by choosing a different contour of integration completely included in the convergence domain of the series (4) (see [12]), namely a contour $C_{r}$ formed with the half circle $\gamma_{r}: z=r \mathrm{e}^{i \theta},-\pi / 2 \leq \theta \leq \pi / 2$, where $r$ is small enough such that no divergence point of (4) except the origin is located on the diameter of this half circle, and the half lines $\operatorname{Im} z= \pm r, \operatorname{Re} z \geq 0$ (see Figure 1 below).

It can be easily checked that $\int_{C_{r}}(-z)^{s-1} \zeta_{A, \mathrm{e}^{\Lambda}}(z) \mathrm{d} z$ does not depend on $r$ and the integral on $\gamma_{r}$ tends to zero as $r \rightarrow 0$.

Indeed, if $C_{r_{1}}$ is the red contour, $C_{r_{2}}$ is the blue one and we form also the contour $C$ colored green, then $\int_{C_{\eta}}(-z)^{s-1} \zeta_{A, \mathrm{e}^{\mathrm{A}}}(z) \mathrm{d} z=\int_{C_{r_{2}}}(-z)^{s-1} \zeta_{A, \mathrm{e}^{\wedge}}(z) \mathrm{d} z$

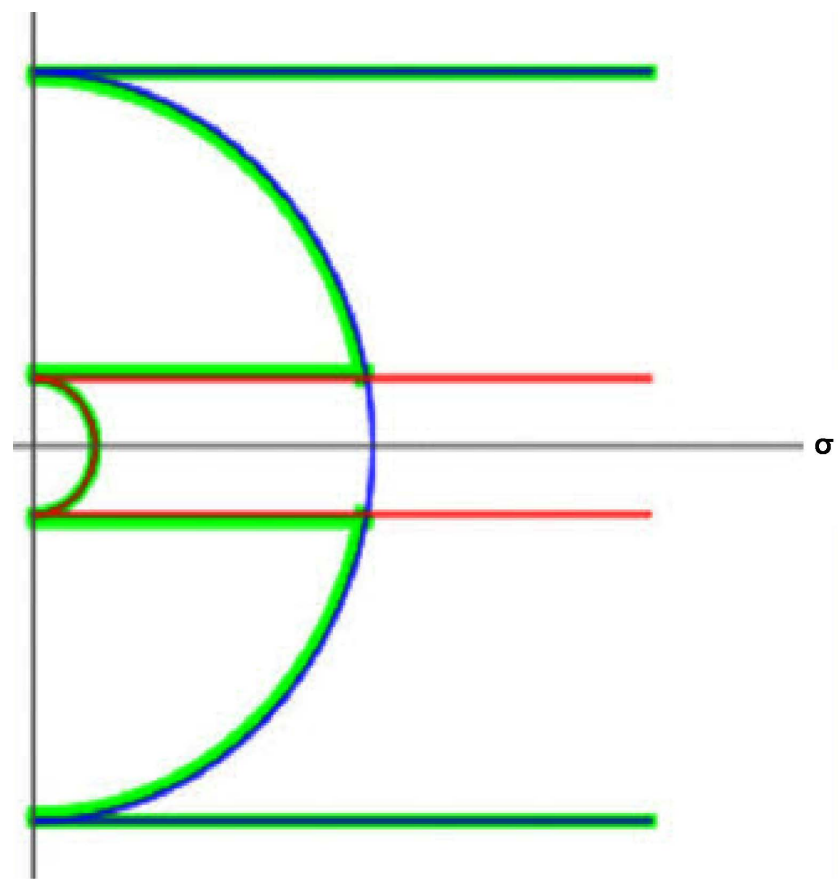

Figure 1. The integral on $C_{r}$ does not depend on $r$. 
since both of these integrals are equal to $\int_{C}(-z)^{s-1} \zeta_{A, \mathrm{e}^{\Lambda}}(z) \mathrm{d} z$.

The fact that the integral on $\gamma_{r}$ tends to zero as $r \rightarrow 0$ can be shown as follows:

$$
\begin{aligned}
& \left|\int_{\gamma_{r}}(-z)^{s-1} \zeta_{A, \mathrm{e}^{\Lambda}}(z) \mathrm{d} z\right| \leq \mathrm{e}^{-\pi t} \sum_{n=1}^{\infty}\left|a_{n}\right| r^{\sigma} \int_{-\pi / 2}^{\pi / 2} \mathrm{e}^{-\left(\theta t+\mathrm{e}^{\lambda_{n}} r \cos (\theta)\right)} \mathrm{d} \theta \\
& =\mathrm{e}^{-\pi t} \sum_{n=1}^{\infty}\left|a_{n}\right| r^{\sigma} \int_{-\pi / 2}^{\pi / 2}\left[1-\left(\theta t+\mathrm{e}^{\lambda_{n}} r \cos (\theta)\right)+\frac{1}{2 !}\left(\theta t+\mathrm{e}^{\lambda_{n}} r \cos (\theta)\right)^{2}+\cdots\right] \mathrm{d} \theta .
\end{aligned}
$$

The series (4) converges absolutely on $\gamma_{r}$, hence when evaluating this integral we are free to deal separately with different groups of terms. The sum of terms under the integral which do not contain $r$ is $1-\theta t+\frac{1}{2 !}(\theta t)^{2}+\cdots=\mathrm{e}^{-\theta t}$ and $\int_{-\pi / 2}^{\pi / 2} \mathrm{e}^{-\theta t} \mathrm{~d} \theta=\frac{2}{t} \operatorname{sh}\left(\frac{\pi t}{2}\right)$, thus $\lim _{r \rightarrow 0} r^{\sigma} \int_{-\pi / 2}^{\pi / 2} \mathrm{e}^{-\theta t} \mathrm{~d} \theta=0$. The terms which contain $r$ under that integral tend all to zero as $r \rightarrow 0$, and therefore their integrals cancel too at the limit. We conclude that:

$$
\lim _{r \rightarrow 0} \int_{\gamma_{r}}(-z)^{s-1} \zeta_{A, \mathrm{e}^{\Lambda}}(z) \mathrm{d} z=0
$$

When $r=0$ the curve $C$ becomes the positive real half axis traversed back and forth. On the upper edge we have $(-z)^{s-1}=x^{s-1} \mathrm{e}^{-(s-1) \pi i}$ and on the lower edge $(-z)^{s-1}=x^{s-1} \mathrm{e}^{(s-1) \pi i}$ and then:

$$
\begin{aligned}
& \int_{C}(-z)^{s-1} \zeta_{A, \Lambda}(z) \mathrm{d} z \\
& =-\int_{0}^{\infty} x^{s-1} \mathrm{e}^{-(s-1) \pi i} \zeta_{A, \mathrm{e}^{\Lambda}}(x) \mathrm{d} x+\int_{0}^{\infty} x^{s-1} \mathrm{e}^{(x-1) \pi i} \zeta_{A, \mathrm{e}^{\Lambda}}(x) \mathrm{d} x \\
& =2 i \sin ((s-1) \pi) \int_{0}^{\infty} x^{s-1} \zeta_{A, \mathrm{e}^{\Lambda}}(x) \mathrm{d} x=-2 i \sin (\pi s) \zeta_{A, \Lambda}(s)
\end{aligned}
$$

Since $\Gamma(s) \Gamma(1-s)=\frac{\pi}{\sin (\pi s)}$ the formula (9) becomes:

$$
\zeta_{A, \Lambda}(s)=-\frac{\Gamma(1-s)}{2 \pi i} \int_{C}(-z)^{s-1} \zeta_{A, e^{\Lambda}}(z) \mathrm{d} z
$$

The limit (8) guarantees that this integral is convergent no matter if $\lim _{x \backslash 0} \zeta_{A, \mathrm{e}^{\wedge}}(x)$ is finite or not and the theorem is completely proved. Obviously, at a point $s$ where the integral does not cancel, $\zeta_{A, \Lambda}(s)$ can have a pole if $\Gamma(1-s)$ has a pole.

Let us examine some Dirichlet series which do not admit meromorphic continuation across the convergence line. The series $\sum_{n=1}^{\infty} \frac{1}{(n !)^{s}}$ has been suggested by Dan Asimov in a private communication. This is an ordinary Dirichlet series, i.e. a series of the form $\sum_{m=1}^{\infty} \frac{a_{m}}{m^{s}}$, where $a_{m}=1$ if $m=n !$ for some $n$ and $a_{m}=0$ otherwise. Let $A$ be this sequence of coefficients and let $\Lambda$ the sequence of exponents be defined by $\lambda_{n}=\ln (n !)$. Then 


$$
\sum_{n=1}^{\infty} \frac{1}{(n !)^{s}}=\sum_{n=1}^{\infty} \mathrm{e}^{-\ln (n !) s}=\zeta_{A, \Lambda}(s)
$$

and

$$
\zeta_{A, \mathrm{e}^{\Lambda}}(s)=\sum_{n=1}^{\infty} \mathrm{e}^{-(n !) s}
$$

Using the formula (2) it can be easily checked that both of these series have the abscissa of convergence 0 , which agrees with the Proposition 1 . A result of Tanaka ([14], Corollary VI) tells us that the series (1) has the imaginary axis as natural boundary if: $\sigma_{c}=0, \liminf _{n \rightarrow \infty}\left(\lambda_{n+1}-\lambda_{n}\right)>0$ and $\lim _{n \rightarrow \infty} n / \lambda_{n}=0$. It is easy to check that these series have both the imaginary axis as natural boundary, hence they cannot be meromorphically continued across the imaginary axis. We can give a direct proof for the series (12), as an easy exercise.

Theorem 2. The series (12) diverges at every point of the imaginary axis.

Proof: Let us notice that $\zeta_{A, \mathrm{e}^{\Lambda}}(s)=\sum_{n=1}^{\infty}\left(\mathrm{e}^{-s}\right)^{n !}$, which is a power series of the form $\sum_{m=1}^{\infty} a_{m} w^{m}$, where $a_{m}=1$ if $m=n$ ! for some $n$ and $a_{m}=0$ otherwise, and $w=\mathrm{e}^{-s}$.

The Hadamard's formula shows that the radius of convergence of this series is 1, i.e. the series converges for $|w|<1$ and it diverges for $|w|>1$. Let us show that the series diverges at every point of the unit circle. Since the set of points $w=\mathrm{e}^{i \theta}$ with the argument $\theta$ rational multiple of $2 \pi$ is dense in the unit circle, it will be enough to show that the series diverges at every one of these points. Suppose $w=\mathrm{e}^{2 \pi i p / q}$, where $p$ and $q$ are integers.

Then, for $0<\rho<1$ we have $\sum_{n=1}^{\infty}(\rho w)^{n !} \geq\left|\sum_{n=q}^{\infty}(\rho w)^{n !}\right|-\left|\sum_{n=1}^{q-1}(\rho w)^{n !}\right|$. Let us notice that $(\rho w)^{n !}=\rho^{n !} \mathrm{e}^{2 \pi i n ! p / q}$, thus for $n \geq q$ we have $(\rho w)^{n !}=\rho^{n !}$, since $n$ ! is a multiple of $q$ and therefore $2 \pi i n ! p / q$ is an integer multiple of $2 \pi i$, hence $w^{n !}=1$. For $n<q$ we have $\left|(\rho w)^{n !}\right|=\rho^{n !}<1$, thus $\left|\sum_{n=1}^{q-1}(\rho w)^{n !}\right|=\sum_{n=1}^{q-1} \rho^{n !}<q$, therefore $\left|\sum_{n=1}^{\infty}(\rho w)^{n !}\right| \geq \sum_{n=q}^{\infty} \rho^{n !}-q \rightarrow \infty$ as $\rho \rightarrow 1$, which means that $\sum_{n=1}^{\infty} w^{n !}$ diverges for $|w|=1$. Yet, $1=|w|=\left|\mathrm{e}^{-(\sigma+i t)}\right|=\mathrm{e}^{-\sigma}$ implies $\sigma=0$, hence the series (12) diverges at every point of the imaginary axis. This means that Theorem 2 cannot be used in this case in order to prove that the series (11) admits a meromorphic continuation into the whole complex plane. However, Tanaka's result confirms that this is not the case.

Numerous other examples can be given of Dirichlet series having the imaginary axis as their natural boundary. If in the formula (11) we take instead of the sequence $A$ a sequence $A_{m}$ depending on an integer $m \geq 2$ such that $a_{n}=1$ if $n=m^{k}$ for some integer $k \geq 1$ and $a_{n}=0$ otherwise, and we chose the exponents $\lambda_{n}=\ln (n)$ then $\zeta_{A_{m}, \Lambda}(s)=\sum_{n=1}^{\infty} \frac{1}{\left(n^{m}\right)^{s}}=\sum_{n=1}^{\infty} \frac{1}{n^{m s}}=\zeta(m s)$ and $\zeta_{A_{m}, \mathrm{e}^{\Lambda}}(s)=\sum_{n=1}^{\infty} \mathrm{e}^{-n^{m} s}=\sum_{n=1}^{\infty}\left(\mathrm{e}^{-s}\right)^{n^{m}}$, for which, by Tanaka's theorem, the imaginary axis is the natural boundary. However, the associated series $\zeta_{A, \Lambda}(s)$ 
admits meromorphic continuation into the whole complex plane.

\section{The Geometry of Mappings by Dirichlet Functions}

We dealt in [15] with different classes of Dirichlet series, all admitting meromorphic continuation into the whole complex plane and we found that the respective Dirichlet functions displayed strong similarities regarding the geometry of their conformal mappings. We will list here those properties for a generic Dirichlet function.

Theorem 3. For any Dirichlet function $\zeta_{A, \Lambda}(s)$ generated by a general Dirichlet series with $\sigma_{a}<\infty$ we have $\lim _{\sigma \rightarrow+\infty} \zeta_{A, \Lambda}(\sigma+i t)=1$ uniformly with respect to $t$.

Proof: Let $\sigma_{a}$ be the abscissa of absolute convergence of $\zeta_{A, \Lambda}(s)$. For any $\sigma_{0}>\sigma_{a}$ and $\sigma>\sigma_{0}$ we have:

$\left|\sum_{n=2}^{\infty} a_{n} \mathrm{e}^{-\lambda_{n} s}\right| \leq \sum_{n=2}^{\infty}\left|a_{n}\right| \mathrm{e}^{-\lambda_{n}\left(\sigma-\sigma_{0}\right)} \mathrm{e}^{-\lambda_{n} \sigma_{0}}$ $=\mathrm{e}^{-\lambda_{2}\left(\sigma-\sigma_{0}\right)} \sum_{n=2}^{\infty}\left|a_{n}\right| \mathrm{e}^{-\left(\lambda_{n}-\lambda_{2}\right)\left(\sigma-\sigma_{0}\right)} \mathrm{e}^{-\lambda_{n} \sigma_{0}} \leq K \mathrm{e}^{-\lambda_{2} \sigma} \rightarrow 0$

as $\sigma \rightarrow+\infty$. Here $K$ is a constant (not depending on $\sigma$ ) given the fact that $\mathrm{e}^{-\left(\lambda_{n}-\lambda_{2}\right)\left(\sigma-\sigma_{0}\right)} \leq 1$, $\mathrm{e}^{\lambda_{2} \sigma_{0}}$ is a constant and $\sum_{n=2}^{\infty}\left|a_{n}\right| \mathrm{e}^{-\lambda_{n} \sigma_{0}}$ is convergent. Hence $\sum_{n=2}^{\infty} a_{n} \mathrm{e}^{-\lambda_{n}(\sigma+i t)}$ converges to zero uniformly with respect to $t$ when $\sigma \rightarrow+\infty$, thus $\lim _{\sigma \rightarrow+\infty} \zeta_{A, \Lambda}(\sigma+i t)=1$ uniformly with respect to $t$. This means that given $\varepsilon>0$, there is $\sigma_{\varepsilon}$ such that for $\sigma>\sigma_{\varepsilon}$ we have $\left|\zeta_{A, \Lambda}(\sigma+i t)-1\right|<\varepsilon$ i.e. the whole half plane $\operatorname{Re} s>\sigma_{\varepsilon}$ is mapped by $z=\zeta_{A, \Lambda}(s)$ into the disc centered at $z=1$ and having the radius $\varepsilon$.

In what follows we will deal only with Dirichlet functions satisfying the condition of Theorem 3. It is obvious that the zeros of such a function are isolated points, since otherwise the function would be identically zero. This implies that for any bounded domain $D$ the components of the pre-image of a circle $|z|=r$ included in $D$ are disjoint closed curves if $r$ is small enough. For such an $r$ the domains bounded by those curves are mapped by $\zeta_{A, \Lambda}(s)$ onto the disc $|z|<r$. The mapping is conformal for those domains containing a simple zero. If the zero is multiple of order $m$, then the pre-image of the radius $0 \leq \operatorname{Re} z \leq 1$ partitions $D$ into $m$ sets whose interior are mapped conformally by $\zeta_{A, \Lambda}(s)$ onto $0<|z|<1$ (see [13], page 133). When increasing $r$, these components expand and some of them can touch each other at points $s_{k}$ or they become disjoint infinite curves. At the points $s_{k}$ we have $\zeta_{A, \Lambda}^{\prime}\left(s_{k}\right)=0$. Moreover, if $\zeta_{A, \Lambda}^{\prime}\left(s_{0}\right)=0$, then $s_{0}$ is either a multiple zero of $\zeta_{A, \Lambda}(s)$ or one of the points $s_{k}$. Figure 2(a) below exhibits two disjoint components of the pre-image by the Riemann Zeta function of a circle $|z|=r<1$. The little one contains one zero of $\zeta(s)$ and the other one contains two zeros. For a bigger value of $r$ those expanded components have met into a point $s_{0}$ where $\zeta^{\prime}\left(s_{0}\right)=0$ as shown in Figure 2(b). Increasing $r$ even more, the component obtained turns around all three zeros of $\zeta(s)$, Figure 2(c). When $r=1$ some components must become unbounded, as stated in the following theorem see also ([16]): 


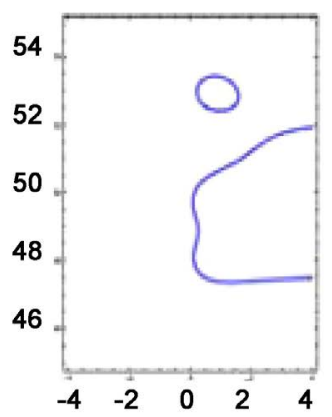

(a)

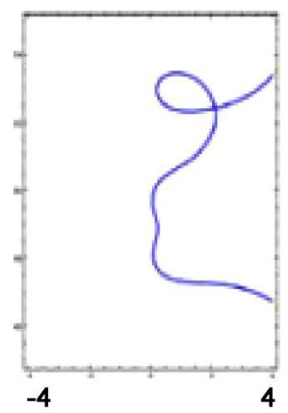

(b)

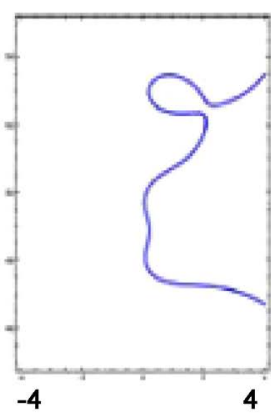

(c)

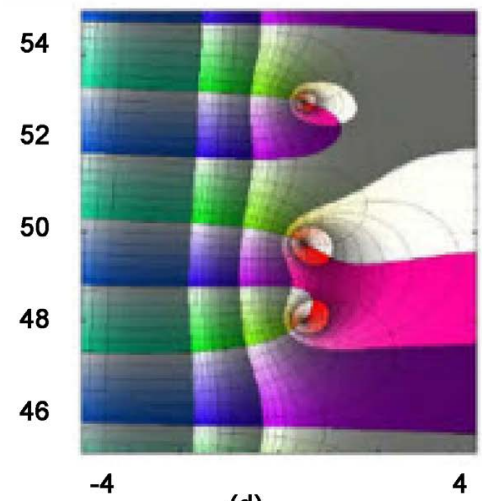

(d)

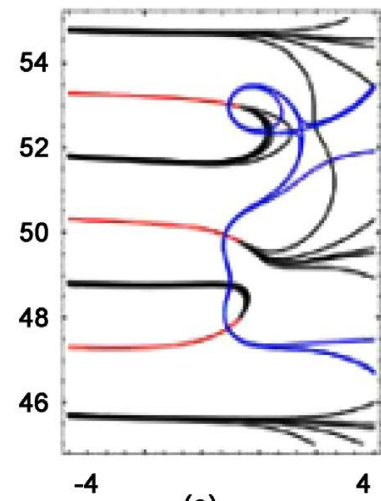

(e)

Figure 2. (a): Components around one zeros and two zeros; (b): The components touch each other; (c): An unique component containing the three zeros; (d): Color visualization of the mapping; (e): The evolution the pre-image of a ray approaching the real axis.

Theorem 4: For every Dirichlet function $\zeta_{A, \Lambda}(s)$ defined by a normalized series (1) the pre-image of the unit disc has at least one unbounded component.

Proof: Assume that all the components of the pre-image of the closed unit disc were bounded. By Theorem 3, for every $\varepsilon>0$, there is $\sigma_{\varepsilon}$ such that the half plane $U_{\varepsilon}=\left\{\sigma+i t \mid \sigma>\sigma_{\varepsilon}\right\}$ is mapped by $\zeta_{A, \Lambda}(s)$ into the disc $|z-1|<\varepsilon$. For arbitrary $\delta>0$, let us denote by $V$ a $\delta$-neighborhood of the pre-image of the closed unit disc. The components of $V$ are bounded connected open sets. Due to the fact that $\zeta_{A, \Lambda}(s)$ is an open mapping, $V$ is mapped by $\zeta_{A, \Lambda}(s)$ onto an open set containing the closed unit disc. If $\varepsilon>0$ is small enough, the closed disc centered at the origin and of radius $1+\varepsilon$ is included in that open set, hence its pre-image is included in $V$. Therefore it can have only bounded connected components. Since $|z-1|<\varepsilon$ implies $|z|<1-\varepsilon$ one of these components should contain the half plane $U_{\varepsilon}$, which is a contradiction. Thus the pre-image of the unit disc must contain at least one unbounded component.

Starting with Speiser's work [17] the pre-image of the real axis has been used in order to describe the geometry of mappings by the Riemann Zeta function.

A historic account of this technique can be found in [16], Section 3. In our works ([12] [15] [16] [18]) we have used a better way to visualize the conformal mapping by an analytic function $z=f(s)$, namely by taking the pre-image of 
an orthogonal mesh from the $z$-plane formed with rays issuing from the origin and circles centered at the origin. A spectre of colors can be superposed to this mesh and care can be taken that every point $s$ in the pre-image plane has the same color as its image $f(s)$. Given a fundamental domain we are then able to see the one-to-one correspondence between the points in the domain and their images. However, distinguishing the fundamental domains of a function is not an easy task, since the partition of the complex plane into fundamental domains is not unique. An illustration of this technique is exhibited in Figure 3 below where $f(s)$ is the Riemann Zeta function.

Given such a partition, a slight deformation of one fundamental domain implies different deformations of all the other domains. The only points which must remain unchanged are the branch points, which are common boundary points of different fundamental domains. They are the zeros of $f^{\prime}(z)$, the multiple poles, as well as the essential singular points. By Big Picard Theorem, every neighborhood of an essential singular point of $f(s)$ intersects infinitely many fundamental domains and it is mapped (not necessarily one-to-one) onto the whole complex plane with one possible point removed.

In what follows we will use the concept of continuation above a curve (see [19], page 28). The Dirichlet functions $\zeta_{A, \Lambda}(s)$ are transcendental functions for which $s=\infty$ is an essential singular point. Let $x_{0}>1$ be an arbitrary positive number $s_{0} \in \mathbb{C}$ such that $\zeta_{A, \Lambda}\left(s_{0}\right)=x_{0}$. The continuation by $\zeta_{A, \Lambda}(s)$ from $s_{0}$ along the interval $(1,+\infty)$ is a curve $\Gamma$ such that when $x=\zeta_{A, \Lambda}(\sigma+i t) \rightarrow 1$

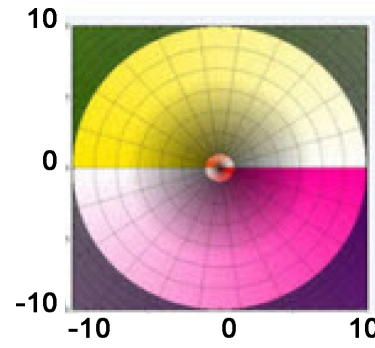

(a)

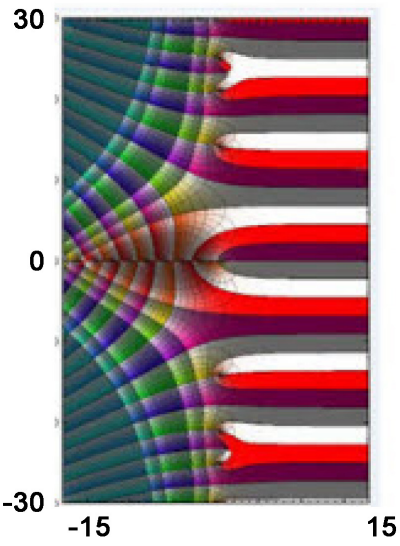

(d)

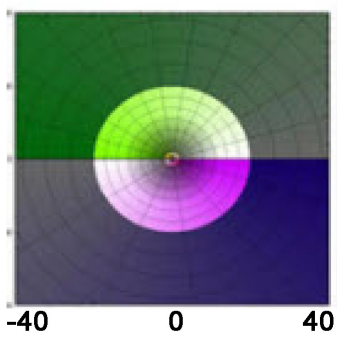

(b)
1020

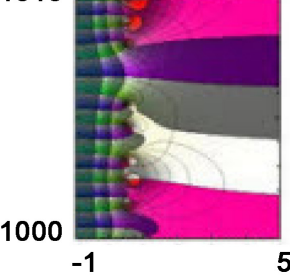

(e)

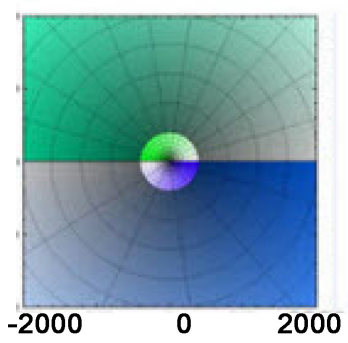

(c)

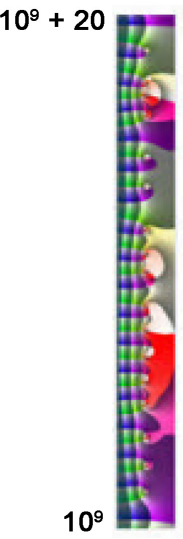

(f)

Figure 3. Color visualization of the conformal mapping by the $\zeta(s)$. 
we have that $\sigma \rightarrow+\infty$ with $s=\sigma+i t$ on $\Gamma$, or there is a point $u \in \Gamma$ such that $\zeta_{A, \Lambda}(u)=1$ and the continuation can be carried along the real axis for values less than 1 . When $s$ does not meet any pole in its way, we have that $s \rightarrow \infty$ on $\Gamma$ when $x \rightarrow-\infty$, i.e. $\Gamma$ is unbounded at both ends and it is mapped one-to-one onto the whole real axis. If $s$ meets a pole, the continuation stops there in the sense that when $x \rightarrow-\infty, s$ approaches that pole.

Let us take a ray $\eta_{\alpha}$ making a small angle $\alpha>0$ with the positive real half axis in the $z$-plane and let $z_{\alpha}$ be the intersection of this ray with the unit circle. The pre-image of $z_{\alpha}$ by $\zeta_{A, \Lambda}(s)$ is a set of points belonging each one to a component of the pre-image of the unit circle. One of them $s_{\alpha}=\sigma_{\alpha}+i t_{\alpha}$ belongs to the unbounded component. The continuation over $\eta_{\alpha}$ from $s_{\alpha}$ is an unbounded curve $\Gamma_{\alpha}$ starting at a zero of $\zeta_{A, \Lambda}(s)$ and such that when $s=\sigma+i t \in \Gamma_{\alpha}$ we have that $\sigma \nrightarrow+\infty$, since $z_{\alpha}=\zeta_{A, \Lambda}(s) \nrightarrow 1$ (see Figure 2(d) and Figure 2(e)). Yet, $\sigma_{\alpha} \rightarrow+\infty$ when $\alpha \rightarrow 0$. The curve $\Gamma_{\alpha}$ has a part $\Gamma_{\alpha}^{\prime}$ above that unbounded component and another part $\Gamma_{\alpha}^{\prime \prime}$ inside it. The first one is unbounded. At the limit, when $\alpha=0, \Gamma_{\alpha}^{\prime \prime}$ becomes also unbounded and it is a part of a component $\Gamma_{k, 0}$ of the pre-image of the real axis which is mapped one-to-one by $\zeta_{A, \Lambda}(s)$ onto the interval $(-\infty, 1)$.

By taking $\alpha<0$ we conclude again that $\Gamma_{\alpha}^{\prime \prime}$ is a part of $\Gamma_{k, 0}$, while $\Gamma_{\alpha}^{\prime}$ is below that unbounded component of the pre-image of the unit circle.

We summarize by saying (see [16]) that there can be three types of connected components of the pre-image of the real axis by $\zeta_{A, \Lambda}(s)$, namely those which are mapped one-to-one onto the interval $(1,+\infty)$, denoted $\Gamma_{k}^{\prime}$, those which are mapped one-to-one onto the interval $(-\infty, 1)$ denoted $\Gamma_{k, 0}$ and finally those which are mapped one-to-one onto the whole real axis, denoted $\Gamma_{k, j}, j \neq 0$.

Proposition 2: There are infinitely many connected components $\Gamma_{k}^{\prime}, k \in \mathbb{Z}$ of the pre-image of the real axis by $\zeta_{A, \Lambda}(s)$. No two such components can intersect each other and consecutive ones $\Gamma_{k}^{\prime}$ and $\Gamma_{k+1}^{\prime}$ form infinite strips $S_{k}$, $0 \in S_{0}$ extending for $\sigma$ from $-\infty$ to $+\infty$ (see [16], Theorem 5), which are mapped by $\zeta_{A, \Lambda}(s)$ (not necessarily one-to-one) onto the whole complex plane. The strips $S_{k}$ cover entirely the complex plane. Every strip $S_{k}, k \neq 0$ contains a unique unbounded component of the pre-image of the unit circle and a unique component $\Gamma_{k, 0}$ of the pre-image of the real axis which is mapped by $\zeta_{A, \Delta}(s)$ one-to-one onto the interval $(-\infty, 1)$. There are in general several components $\Gamma_{k, j}, j \neq 0$ in every strip $S_{k}$ which are mapped one-to-one onto the whole real axis. Obviously, every $\Gamma_{k, j}$ must contain a zero of $\zeta_{A, \Lambda}(s)$. The strip $S_{0}$ can contain infinitely many curves $\Gamma_{0, j}$.

All these affirmations are proved in Section 3 of [13]. An illustration of them is provided in Figure 4 below for two Dirichlet L-functions, one defined by a real Dirichlet character and the other by a complex one.

Every strip $S_{k}$ can be partitioned into sub-strips, which are fundamental domains of $\zeta_{A, \Lambda}(s)$, i.e. which are mapped conformally by $\zeta_{A, \Lambda}(s)$ onto the whole complex plane with some slits. For example, if the strip $S_{k}$ contains a 


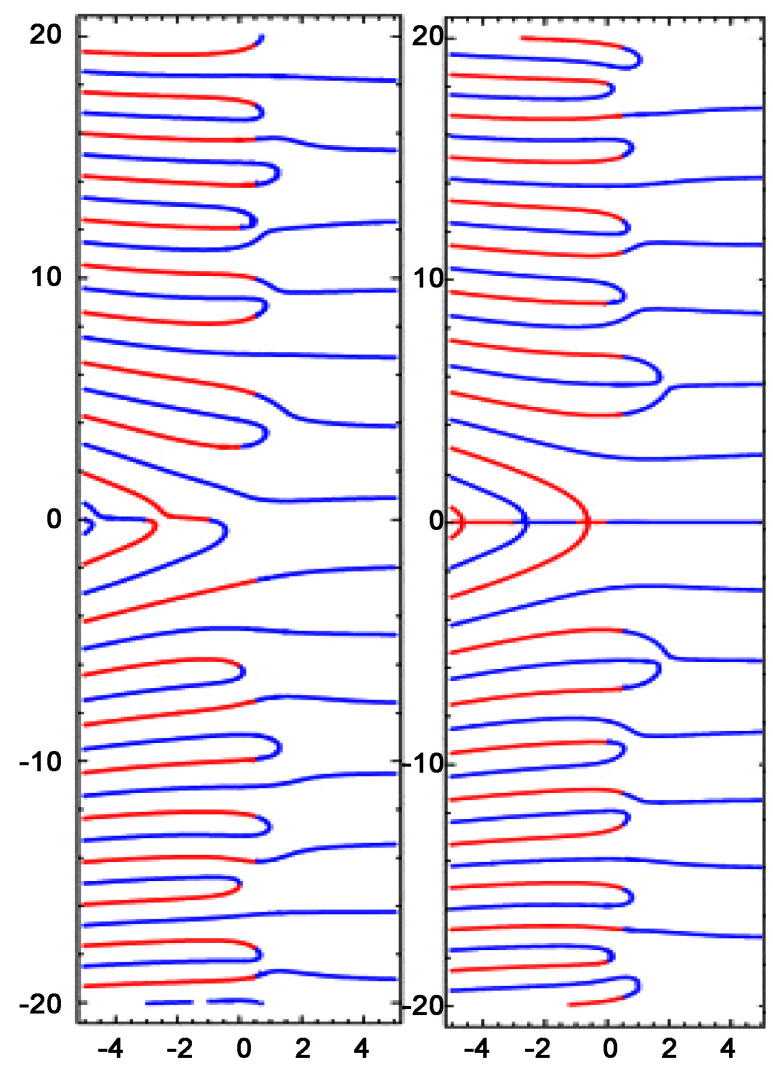

Figure 4. The pre-image of the real axis by two Dirichlet L-functions.

zero $v_{k, j}$ of $\zeta_{A, \Lambda}^{\prime}(s)$, then the pre-image of the segment $I_{k, j}$ from $z=1$ to $z=\zeta_{A, \Lambda}\left(v_{k, j}\right)$ included in $S_{k}$ is formed with either disjoint arcs $\eta_{k, j}$ originating in points $u_{k, j}$ on curves $\Gamma_{k, j}$ with $\zeta_{A, \Lambda}\left(u_{k, j}\right)=1$ and ending in some points $u_{k j^{\prime}}$ on $\Gamma_{k, j^{\prime}}$ such that $\zeta_{A, \Lambda}\left(u_{k, j^{\prime}}\right)=1$, or with disjoint unbounded curves $\eta_{k, j}$ originating in $u_{k, j}$ and such that when $z$ on $I_{k, j}$ approaches 1, the corresponding point $s=\sigma+i t \in \eta_{k, j}$ is such that $\sigma \rightarrow+\infty$. These arcs or unbounded curves are mapped two to one onto the segment $I_{k, j}$. Together with the pre-image of the interval $(1,+\infty)$ they bound domains which are mapped conformally by $\zeta_{A, \Lambda}(s)$ onto the whole complex plane with a slit alongside the interval $[1,+\infty)$ followed by $I_{k, j}$. We get arcs $\eta_{k, j}$ in the case of embraced curves as shown in Figure 5 below, and also in the case of $S_{0}$ where any two consecutive curves $\Gamma_{0, j}$ and $\Gamma_{0, j+1}$ are embraced (see Figure 4 above). In all the other cases the curves $\eta_{k, j}$ are unbounded. There is a one-to-one correspondence between the curves $\Gamma_{k, j}, j \neq 0$ from every strip $S_{k}$ and the zeros of $\zeta_{A, \Lambda}^{\prime}(s)$ from that strip (see [16], Theorem 6), which makes possible the construction of the arcs and curves $\eta_{k, j}$ and, as a result, that of the fundamental domains of $\zeta_{A, \Lambda}(s)$ included in that strip.

\section{The Zeros of Dirichlet Functions}

It is obvious that every curve $\Gamma_{k, j}$ contains a unique zero, since $\Gamma_{k, j}$ is projected 


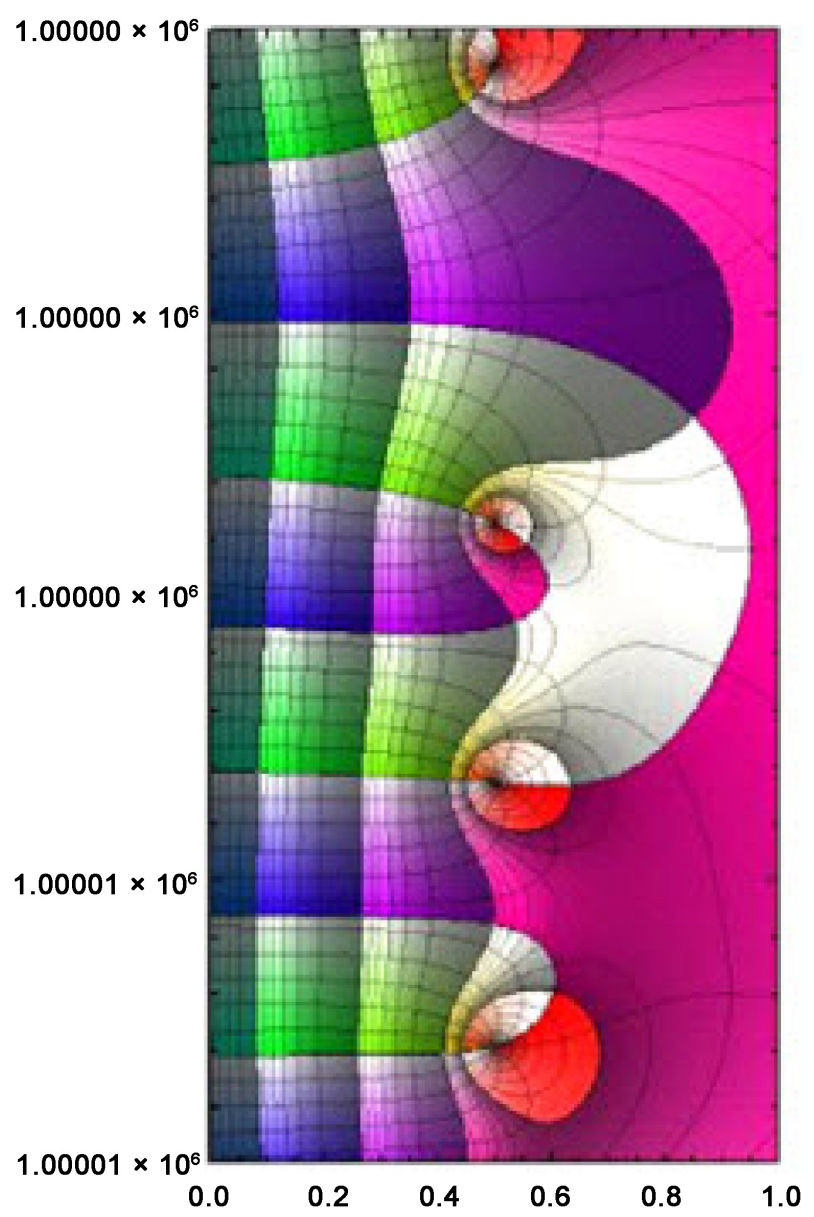

Figure 5. Embraced curves for $\zeta$ function in the range of $t=1.00002 \times 10^{6}$.

one to one onto the real axis if $j \neq 0$ and onto the interval $(-\infty, 1)$ if $j=0$. Moreover, no zero can exist outside these curves and if a zero $s_{0}$ has the order of multiplicity $m$ then $m$ curves $\Gamma_{k, j}$ must pass through $s_{0}$. We have shown (see [15], Theorem 3) that $m \leq 2$ and every strip $S_{k}$ can contain at most one double zero. When such a zero exists it must be at the intersection of $\Gamma_{k, 0}$ with $\Gamma_{k,-1}$ or with $\Gamma_{k, 1}$. Examples of double zeros have been obtained in [15] by taking linear combinations of Dirichlet L-functions and by using the fact that those functions satisfy Riemann type of functional equations. There are a lot of other classes of Dirichlet functions satisfying Riemann type of functional equations and Selberg [20] revealed their connection with the Riemann Hypothesis. In his axiomatic approach, dealing with ordinary Dirichlet series, another property that those series can have has been taken into account, namely of being expressible as an Euler product. We continue to deal with general Dirichlet series which admit meromorphic extensions into the whole complex plane and look for properties of the respective functions similar to those postulated by Selberg.

Let us notice first that the functional equation Riemann has found for the $\zeta$ function has been a by-product of the continuation process of the $\zeta$-series 
through contour integration (see [13], page 216). By a happy coincidence, the sum of residues of the integrand has as a factor $\zeta(1-s)$. The other factor is a trigonometric function whose zeros are easily computed. They are trivial in Riemann's view. The Riemann Hypothesis $(\mathrm{RH})$ concerns the other zeros, the non trivial ones. For them $\zeta\left(s_{0}\right)=0$ if and only if $\zeta\left(1-\overline{s_{0}}\right)=0$, which means that either $\operatorname{Re} s_{0}=1 / 2=\operatorname{Re}\left(1-\overline{s_{0}}\right)$, hence we have a unique zero on the critical line $\operatorname{Re} s=1 / 2$, or we have two zeros symmetric with respect to the critical line. The RH says that for every non trivial zero $s_{0}$ of $\zeta(s)$ we have $s_{0}=1-\bar{s}_{0}$ i.e. $\operatorname{Re} s_{0}=1 / 2$. We will assume that $\zeta_{A, \Lambda}(s)$ satisfies a Riemann type of functional equation, i.e. $\zeta_{A, \Lambda}(s)=M(s) \overline{\zeta_{A, \Lambda}(1-\bar{s})}$, where the multiplier $M(s)$ is a meromorphic function in the complex plane whose zeros can be trivially computed. We will call the zeros of $M(s)$ trivial zeros of $\zeta_{A, \Lambda}(s)$ and we notice that the off critical line non trivial zeros of $\zeta_{A, \Lambda}(s)$ are symmetric with respect to the critical line. All the functions of the Selberg class satisfy this property. However there are a lot of other functions satisfying a similar property. For example, it is known that for the Riemann alternate Zeta function $\zeta_{a}(s)=1-1 / 2^{s}+1 / 3^{s}-\cdots$ we have $\zeta_{a}(s)=\left(1-1 / 2^{s-1}\right) \zeta(s)$ and since $\zeta(s)=2^{s} \pi^{s-1} \sin \left(\frac{\pi s}{2}\right) \Gamma(1-s) \zeta(1-s)$ we obtain

$\zeta_{a}(s)=\left[\left(2^{s}-2\right) /\left(1-1 / 2^{s}\right)\right] \pi^{s-1} \sin \left(\frac{\pi s}{2}\right) \Gamma(1-s) \zeta_{a}(1-s)$. It is obvious that besides the trivial zeros of $\zeta(s)$, the function $\zeta_{a}(s)$ has the trivial zeros $(1+2 k \pi i) / \ln 2, k \in \mathbb{Z}$. The non-trivial zeros of $\zeta(s)$ and $\zeta_{a}(s)$ coincide. Analogously (see [16]), for any modulus $q>1$ the Dirichlet L-function defined by the principal character modulo $q$, which is $L(q, 1, s)=\left(1-q^{-s}\right) \zeta(s)$ has as trivial zeros besides those of $\zeta(s)$ the points $2 k \pi i / \ln q, k \in \mathbb{Z}$. Also, if $\chi$ is an imprimitive Dirichlet character modulo $q$ induced by a primitive Dirichlet character $\chi_{1}$ modulo $q_{1}$, then $L(s, \chi)=L\left(s, \chi_{1}\right) \Pi_{p \mid q}\left[1-\chi_{1}(p) p^{-s}\right]$ and we see that the non-trivial zeros of $L(s, \chi)$ and $L\left(s, \chi_{1}\right)$ are the same, since the zeros of the product are trivial. We believe that this is what Riemann meant by trivial zeros of $\zeta(s)$ and not the fact that they are real zeros. It is just a coincidence that in the particular case of $\zeta(s)$ they are the same. This is a crucial fact to be settled, since the extension of $\mathrm{RH}$ depends essentially on it. We have proved in [16]:

Proposition 3: If $\zeta_{A, \Lambda}(s)$ satisfies a Riemann type of functional equation, but does not satisfy the RH, then:

(a) The off critical line non-trivial zeros appear in couples of the form $s_{1}=\sigma_{1}+i t$ and $s_{2}=\sigma_{2}+i t=1-\sigma_{1}+i t$, where $\sigma_{1}>1 / 2$. There is $\tau_{0}, 0<\tau_{0}<1$ such that $\zeta_{A, \Lambda}^{\prime}\left(s\left(\tau_{0}\right)\right)=0$, where $s(\tau)=(1-\tau) s_{1}+\tau s_{2}$

(b) $\operatorname{Re} s\left(\tau_{0}\right)<1 / 2$

This affirmation is illustrated in Figure 6 below. Here $s_{0}=s\left(\tau_{0}\right)$ is a branch point for $\zeta_{A, \Lambda}(s)$. Two orthogonal curves intersecting at $s_{0}$ are mapped one to one by $\zeta_{A, \Lambda}(s)$ onto the ray issuing from the origin and passing through $\zeta_{A, \Lambda}\left(s_{0}\right)$. If we ignore the parts of these curves which are projected onto the 


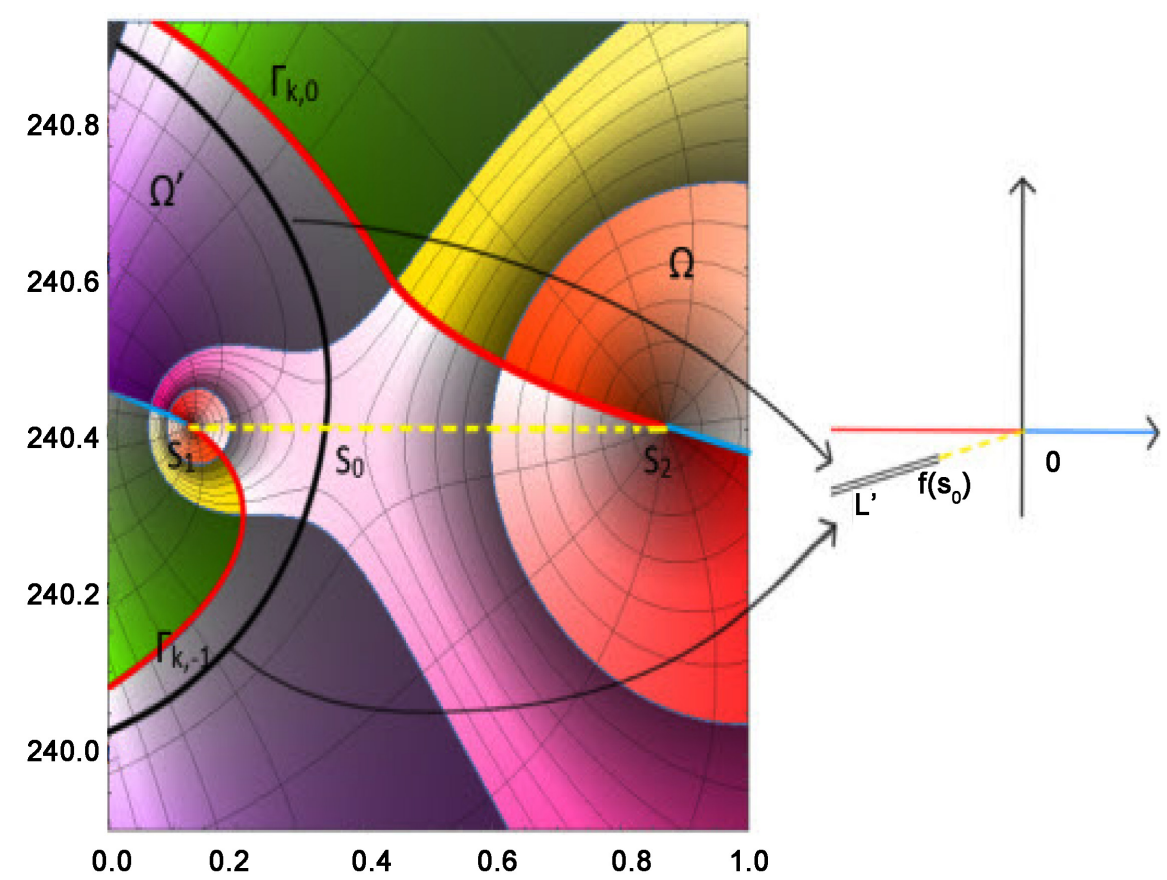

Figure 6. The non-trivial zeros of a Dirichlet function satisfying a Riemann type of functional equation are symmetric with respect to the critical line.

interval from 0 to $\zeta_{A, \Lambda}\left(s_{0}\right)$ (the yellow parts), what remains is an unbounded curve $\gamma$ separating $s_{1}$ and $s_{2}$. This curve is mapped two-to-one onto the part of the ray from $\zeta_{A, \Lambda}\left(s_{0}\right)$ (marked $f\left(s_{0}\right)$ on Figure 6) to infinite and therefore it is a part of the boundaries of the fundamental domains $\Omega_{1}$ and $\Omega_{2}$ which contain respectively $s_{1}$ and $s_{2}$. The conformal mapping

$$
\varphi(s)=\left.\zeta_{A, \Lambda}^{-1}\right|_{\Omega_{2}} \circ \zeta_{A, \Lambda}(s)
$$

of $\Omega_{1}$ onto $\Omega_{2}$ can be extended to an involution of $\Omega_{1} \cup \Omega_{2} \cup \gamma$ in which $\gamma$ is mapped one to one onto itself with the fix point $s_{0}$.

In what follows we will deal with general Dirichlet series whose coefficients and exponents satisfy some special conditions. We consider the coefficients as being the values of arithmetic functions: $\chi: \mathbb{N} \rightarrow \mathbb{C}$ defined by $\chi(n)=a_{n}$. We say that the function $\chi$ is totally multiplicative if $\chi(1)=1$ and for every $m$ and $n$ we have $\chi(m n)=\chi(m) \chi(n)$. The Dirichlet characters are totally multiplicative arithmetic functions defined on $\mathbb{Z}$ for which there exists a positive integer $m$ such that $\chi(n+m)=\chi(n)$ for all $n \in \mathbb{Z}$. We say that $\chi$ is a Dirichlet character modulo $m$. The ordinary Dirichlet series defined by such coefficients are called Dirichlet L-series. We notice that if $\chi$ is totally multiplicative and $n$ has the prime decomposition $n=p_{1}^{\alpha_{1}} p_{2}^{\alpha_{2}} \cdots p_{k}^{\alpha_{k}}$ then

$$
\chi(n)=\chi\left(p_{1}\right)^{\alpha_{1}} \chi\left(p_{2}\right)^{\alpha_{2}} \cdots \chi\left(p_{k}\right)^{\alpha_{k}}
$$

The exponents can be considered also as generated by an arithmetic function $\lambda: \mathbb{N} \rightarrow \mathbb{R}_{+}$by $\lambda(n)=\lambda_{n}$ We say that $\lambda$ is an additive function if $\lambda(m n)=\lambda(m)+\lambda(n)$ for every $m$ and $n$. It can be easily checked that if $\lambda$ is 
additive then for any $n$ with prime decomposition $n=p_{1}^{\alpha_{1}} p_{2}^{\alpha_{2}} \cdots p_{k}^{\alpha_{k}}$ we have

$$
\lambda(n)=a_{1} \lambda\left(p_{1}\right)+\alpha_{2} \lambda\left(p_{2}\right)+\cdots+\alpha_{k} \lambda\left(p_{k}\right) .
$$

Theorem 5. Suppose that the general Dirichlet series

$$
\zeta_{A, \Lambda}(s)=\sum_{n=1}^{\infty} \chi(n) \mathrm{e}^{-\lambda(n)) s}=1+\chi(2) \mathrm{e}^{-\lambda(2) s}+\chi(3) \mathrm{e}^{-\lambda(3) s}+\cdots
$$

converges absolutely for some $s$ and is such that $\chi(n)$ is totally multiplicative and $\lambda(n)$ is additive. Then

$$
\zeta_{A, \Lambda}(s)=\prod_{p \in \wp}\left[1-\chi(p) \mathrm{e}^{-\lambda(p) s}\right]^{-1}
$$

where $\wp$ is the set of prime numbers.

Proof: On multiplying both terms in (14) by $1-\chi(2) \mathrm{e}^{-\lambda(2) s}$ we get

$$
\left[1-\chi(2) \mathrm{e}^{-\lambda(2) s}\right] \zeta_{A, \Lambda}(s)=1+\chi(3) \mathrm{e}^{-\lambda(3) s}+\chi(5) \mathrm{e}^{-\lambda(5) s}+\cdots
$$

where in the right hand side all the terms with even arguments have cancelled since $\chi(2) \chi(k)=\chi(2 k)$ and $\mathrm{e}^{-\lambda(2) s} \mathrm{e}^{-\lambda(k) s}=\mathrm{e}^{-[\lambda(2)+l(k)] s}=\mathrm{e}^{-\lambda(2 k) s}$. If we now multiply in (16) by $1-\chi(3) \mathrm{e}^{-\lambda(3) s}$ we get

$\left[1-\chi(2) \mathrm{e}^{-\lambda(2) s}\right]\left[1-\chi(3) \mathrm{e}^{-\lambda(3) s}\right] \zeta_{A, \Lambda}(s)=1+\chi(5) \mathrm{e}^{-\lambda(5) s}+\chi(7) \mathrm{e}^{-\lambda(7) s}+\cdots$

where in the right hand side all the terms with arguments multiple of 2 and 3 have cancelled. After $n-1$ steps in which we have used consecutive primes $p_{1}, p_{2}, \cdots, p_{n-1}$ the right hand side becomes $1+\chi\left(p_{n}\right) \mathrm{e}^{-\lambda\left(p_{n}\right) s}+\cdots$ all the other coefficients and exponents depending on arguments which are not multiple of $p_{1}, p_{2}, \cdots, p_{n-1}$.

Let us take $\sigma=\operatorname{Re} s>\sigma_{0}>\sigma_{a}$ where $\sigma_{a}$ is the abscissa of absolute convergence of the series (14). By using the notation $\Sigma^{\prime}$ for the summation of terms with arguments not multiple of $p_{1}, p_{2}, \cdots, p_{n-1}$ we have:

$$
\begin{aligned}
\left|\sum_{k \geq p_{n}}^{\prime} \chi(k) \mathrm{e}^{-\lambda(k) s}\right| & \leq \sum_{k \geq p_{n}}^{\prime}|\chi(k)| \mathrm{e}^{-\lambda(k) \sigma} \\
& =\sum_{k \geq p_{n}}^{\prime}|\chi(k)| \mathrm{e}^{-\lambda(k)\left(\sigma-\sigma_{0}\right)} \mathrm{e}^{-\lambda(k) \sigma_{0}} \\
& =\mathrm{e}^{-\lambda\left(p_{n}\right)\left(\sigma-\sigma_{0}\right)} \Sigma_{k \geq p_{n}}^{\prime}|\chi(k)| \mathrm{e}^{-\left[\lambda(k)-\lambda\left(p_{n}\right)\right]\left(\sigma-\sigma_{0}\right)} \mathrm{e}^{-\lambda(k) \sigma_{0}} \\
& \leq \sum_{k=p_{n}}^{\infty}|\chi(k)| \mathrm{e}^{-\lambda(k) s_{0}} \rightarrow 0
\end{aligned}
$$

as $n \rightarrow \infty$, since the series $\sum_{k=1}^{\infty}|\chi(k)| \mathrm{e}^{-\lambda(k) \sigma_{0}} \quad$ is convergent. The last inequality is true because $\mathrm{e}^{-\lambda\left(p_{n}\right)\left(\sigma-\sigma_{0}\right)}<1$ and $\mathrm{e}^{-\left[\lambda(k)-\lambda\left(p_{n}\right)\right]\left(\sigma-\sigma_{0}\right)} \leq 1$ for $k \geq p_{n}$. This shows that the formula (15) is true.

We say that the series (14) is an Euler product series. When it can be continued as a meromorphic function into the whole complex plane we will say that the respective function is an Euler product function. It can be easily checked that the Dirichlet $L$-functions are Euler product functions. On the other hand, totally multiplicative functions can be built by taking $\chi: \wp \rightarrow \mathbb{C}$ arbitrary and extending it to $\mathbb{N}$ by the formula $\left(^{*}\right)$. Obviously, any logarithm function is an additive function, yet the class of additive functions is much larger. Indeed, we can define an arbitrary additive function as follows. Take $\lambda: \wp \rightarrow \mathbb{R}_{+}$and extend it 
to $\mathbb{N}$ by the formula $\left({ }^{*}\right)$ in the following way. First, $\lambda_{1}=0$ and then $\lambda_{2}$ and $\lambda_{3}$ are arbitrary subject to the conditions $0<\frac{3}{2} \lambda_{2}<\lambda_{3}<2 \lambda_{2}$. Then set $\lambda_{4}=2 \lambda_{2}$. Next, take $\lambda_{5}$ arbitrary, subject to the only condition $\lambda_{4}<\lambda_{5}<\lambda_{2}+\lambda_{3}$ and set $\lambda_{6}=\lambda_{2}+\lambda_{3}$. Then take $\lambda_{7}$ arbitrary subject to the condition $\lambda_{6}<\lambda_{7}<3 \lambda_{2}$ and set $\lambda_{8}=3 \lambda_{2}$, and $\lambda_{9}=2 \lambda_{3}$. By the initial inequalities we made sure that $\lambda_{8}<\lambda_{9}$, etc. We can do the same for any two consecutive subscripts and then the function $\lambda: \mathbb{N} \rightarrow \mathbb{R}_{+}$is increasing and the equation (**) is satisfied. With the two functions $\chi$ and $\lambda$ we build arbitrary Euler product general Dirichlet series.

Let us denote by $\mathscr{L}$ the class of functions obtained by meromorphic continuation to the whole complex plane of the series (1) which satisfy a Riemann type of functional equation and are Euler product functions. We suppose also that the series (1) has the abscissa of convergence $\sigma_{c} \leq 0$. The off critical line non-trivial zeros of these functions come in couples $s_{1}=\sigma_{1}+i t_{0}$ and $s_{2}=\sigma_{2}+i t_{0}$, where $\sigma_{2}=1-\sigma_{1}$ and $\sigma_{1} \geq 1 / 2$.

What follows is a new approach to the problem of the location of non-trivial zeros of functions of the class $\mathscr{L}$ undertaken in [21], Theorem 3 and [16], Theorem 13.

Theorem 6: The functions of the class $\mathscr{L}$ satisfy the Riemann Hypothesis, i.e. for every non-trivial zero $s=\sigma+i$ we have $\sigma=1 / 2$.

Proof: Let us denote for the $n$-th prime number $p_{n}$ and any $p \in \wp$ :

$$
f_{n}(s)=\prod_{p \leq p_{n}}\left[1-a_{p} \mathrm{e}^{-\lambda_{p} s}\right]
$$

and notice that $f_{n}(s)$ are integer functions with $\lim _{n \rightarrow \infty} f_{n}(s)=1 / \zeta_{A, \Lambda}(s)$ uniformly on compact sets of the half plane of convergence. Since $\sigma_{c} \leq 0$, a neighborhood of the fix point $s_{0}$ from Figure 5 is mapped onto itself by the function $\varphi(s)$ given by the formula (13) such that for every $s$ in that neighborhood $\zeta_{A, \Lambda}(\varphi(s))=\zeta_{A, \Lambda}(s)$. Then

$$
\lim _{n \rightarrow \infty} f_{n}(\varphi(s)) / f_{n}(s)=\zeta_{A, \Lambda}(s) / \zeta_{A, \Lambda}(\varphi(s)) \equiv 1
$$

in that neighborhood. There is a unique extension of this last ratio to an analytic function in the domain $\Omega_{1} \cup \Omega_{2} \cup \gamma$ of the Proposition 3. The functions $f_{n}(\varphi(s))$ and $f_{n}(s)$ are also defined in that domain. By the permanence of functional equations (see [19], page 288), the Equality (19) must be valid also in $\Omega_{1} \cup \Omega_{2} \cup \gamma$, in particular at $\sigma_{1}+i t_{0}$ and $\sigma_{2}+i t_{0}$.

However, $\zeta_{A, \Lambda}\left(\sigma_{1}+i t_{0}\right)=\zeta_{A, \Lambda}\left(\sigma_{2}+i t_{0}\right)=0$ and therefore their ratio is not defined. Yet, the two zeros have the same order of multiplicity and the l'Hospital rule applies.

The conclusion is that $\zeta_{A, \Lambda}(s) / \zeta_{A, \Lambda}(\varphi(s))$ is identically equal to 1 in $\Omega_{1} \cup \Omega_{2} \cup \gamma$. In particular

$\lim _{n \rightarrow \infty} f_{n}\left(\varphi\left(\sigma_{1}+i t_{0}\right)\right) / f_{n}\left(\sigma_{1}+i t_{0}\right)=1=\zeta_{A, \Lambda}\left(\sigma_{1}+i t_{0}\right) / \zeta_{A, \Lambda}\left(\sigma_{2}+i t_{0}\right)$. Вy (18), this limit is: 


$$
\begin{aligned}
& \lim _{n \rightarrow \infty} \prod_{p \leq p_{n}}\left[\left(1-a_{p} \mathrm{e}^{-\lambda_{p}\left(1-\sigma_{1}+i t_{0}\right)}\right) /\left(1-a_{p} \mathrm{e}^{-\lambda_{p}\left(\sigma_{1}+i t_{0}\right)}\right)\right] \\
& =\lim _{n \rightarrow \infty}\left\{a_{p_{1}} a_{p_{2}} \cdots a_{p_{n}} \exp \left[-\left(\lambda_{p_{1}}+\lambda_{p_{2}}+\cdots+\lambda_{p_{n}}\right)\left(1-\sigma_{1}+i t_{0}\right)\right]+\cdots\right\} \\
& \quad /\left\{a_{p_{1}} a_{p_{2}} \cdots a_{p_{n}} \exp \left[-\left(\lambda_{p_{1}}+\lambda_{p_{2}}+\cdots+\lambda_{p_{n}}\right)\left(\sigma_{1}+i t_{0}\right)\right]+\cdots\right\}
\end{aligned}
$$

The ratio of the leading terms is

$\exp \left[\left(\lambda_{p_{1}}+\lambda_{p_{2}}+\cdots+\lambda_{p_{n}}\right)\left(2 \sigma_{1}-1\right)\right]$ and at the limit as $n \rightarrow \infty$ it should be (19). Yet the limit is $\infty$ if $\sigma_{1}>1 / 2$. Therefore, since (19) is true, we must have $\sigma_{1}=1 / 2$, in which case the ratio of the leading terms in (19) is 1 and therefore the limit is 1 , as expected. Then we have also $\sigma_{2}=1-\sigma_{1}=1 / 2$. This doesn't mean that we have necessarily a double zero. However, if a double zero exists, it should be on the critical line, which agrees with Theorem 6. On the other hand, since simple zeros off critical line cannot exist, $\mathrm{RH}$ for the class $\mathscr{L}$ is completely proved.

\section{Conclusion}

In order to properly tackle the extension of the Riemann Hypothesis to a class of functions generated by general Dirichlet series, we needed to reformulate the concept of trivial and non-trivial zeros. This fact required to redefine the concept of multiplier in a Riemann type of functional equation as a meromorphic function in the complex plane whose zeros can be trivially found. Therefore the multiplier is not necessarily a real function, as usually thought of, and a Riemann type of functional equation in our meaning is more general. What remains the same is the fact that the off critical line non-trivial zeros of a Dirichlet function satisfying a Riemann type of functional equation appear in couples symmetric with respect to the critical line $\operatorname{Re} s=1 / 2$. For the functions derived from Euler product series, it is shown that those zeros must coincide. We used as a tool in proving this assertion known facts about the geometry of mappings by Dirichlet functions, as well as the classic theorem of permanence of functional equations.

\section{Acknowledgements}

We thank Aneta Costin for her support with technical matters.

\section{Conflicts of Interest}

The author declares no conflicts of interest regarding the publication of this paper.

\section{References}

[1] Cahen, E. (1894) Sur la fonction $\zeta(s)$ de Riemann et sur les fonctions analogues. Annales scientifiques de IÉcole Normale Supérieure, Série 3, 11, 75-164. https://doi.org/10.24033/asens.401

[2] Cahen, E. (1918) Sur les séries de Dirichlet. Compte rendue, 166, 987-992.

[3] Hadamard, M.J. (1908) Sur les séries de Dirichlet (Extrait d'une Lettre adressée au 
Directeur dei Rendiconti). Rendiconti del Circolo Matematico di Palermo, 25, 326-330. https://doi.org/10.1007/BF03029133

[4] Landau, E. (1907) Über die Multiplication Dirichletsche Reihen. Rendiconti del Circolo Matematico di Palermo, 24, 81-160. https://doi.org/10.1007/BF03015055

[5] Landau, E. (1909) Über die Konvergenzproblem der Dirichletsche Reihen. Rendiconti del Circolo Matematico di Palermo, 28, 113-151. https://doi.org/10.1007/BF03018214

[6] Bohr, H. (1913) Über die gleichmessiger Konvergenz Dirichletscher Reihen. J. für Math, 143, 203-211. https://doi.org/10.1515/crll.1913.143.203

[7] Bohr, H. (1913) Einige bemerkungen über das Konvergenzproblem Dirichletscher Reihen. Rendiconti del Circolo Matematico di Palermo, 37, 1-16. https://doi.org/10.1007/BF03014810

[8] Bohr, H. (1918) Zür Theorie der allgemeinen Dirichletshe Reihen. Mathematische Annalen, 79, 136-156. https://doi.org/10.1007/BF01457178

[9] Hardi, G.H. and Riesz, M. (1915) The General Theorie of Dirichlet's Series. Cambridge University Press, Cambridge.

[10] Kojima, T. (1914) On the Convergence Abscissa of General Dirichlet Series. Tohoku Mathematical Journal, First Series, 6, 134-139.

[11] Valiron, G. (1926) Théorie générale des séries de Dirichlet. Mémorial des Sciences Mathématiques, 17, 1-56.

[12] Ghisa, D. and Horvat-Marc, A. (2018) Geometric Aspects of Quasi-Periodic Property of Dirichlet Functions. Advances in Pure Mathematics, 8, 699-710. https://doi.org/10.4236/apm.2018.88042

[13] Ahlfors, L.V. (1979) Complex Analysis, International Series in Pure and Applied Mathematics.

[14] Tanaka, C. (1951) Note on Dirichlet Series (1), On the Singularities of the Dirichlet Series (1). Tohoku Mathematical Journal, 3, 285-291.

https://doi.org/10.2748/tmj/1178245483

[15] Ghisa, D. (2017) The Geometry of the Mappings by General Dirichlet Series. Advances in Pure Mathematics, 7, 1-20. https://doi.org/10.4236/apm.2017.71001

[16] Ghisa, D. (2019) Fundamental Domains of Dirichlet Functions. Geometry. Integrability and Quantization, 20, 131-160.

[17] Speiser, A. (1934) Geometrishes zür Riemannschen Zetafunction. Mathematische Annalen, 110, 514-521. https://doi.org/10.1007/BF01448042

[18] Ghisa, D. (2019) On the Zeros of Euler Product Dirichlet Functions. Advances in Pure Mathematics, 9, 959-966. https://doi.org/10.4236/apm.2019.912048

[19] Ahlfors, L.V. and Sario, L. (1960) Riemann Surfaces. Princeton University Press, Princeton. https://doi.org/10.1515/9781400874538

[20] Selberg, A. (1992) Old and New Conjectures and Results about a Class of Dirichlet Series. In: Bompieri, E., et al., Eds., Proceedings of the Amalfi Conference on Analytic Number Theory, Maiori, 367-385.

[21] Ghisa, D. (2016) On Generalized Riemann Hypothesis II. International Journal of Scientific and Innovative Mathematical Research, 4, 46-55.

https://doi.org/10.20431/2347-3142.0403006 